\title{
HARNACK INEQUALITIES FOR EXOTIC BROWNIAN MOTIONS
}

\author{
Hirofumi OSADA \\ (Received 10 May 2001)
}

\begin{abstract}
Exotic Brownian motions are diffusion processes given by Dirichlet forms $\mathcal{E}$ on $L^{2}(\mathbb{S}, \mu)$, where the state space $\mathbb{S}$ is the support of a Radon measure $\mu$ in $\mathbb{R}^{d}$ and the energy form $\mathcal{E}$ is given by the integral of the canonical square field on $\mathbb{R}^{d}$ with respect to $\mu$. In most cases we take $\mu$ in such a way that $\mu$ is singular to the $d$-dimensional Lebesgue measure and, in particular, $\mu$ does not satisfy the doubling condition. The purpose of this paper is to prove the parabolic Harnack inequalities for these diffusions. We will do this with a refinement such that the dependence of $\mu$, Poincaré and Sobolev constants is clarified. Because of the singularity of $\mu$, diffusions are expected to behave in rather an unusual fashion. These Harnack inequalities will be used to show exotic properties of our diffusion processes in a forthcoming paper.
\end{abstract}

\section{Introduction}

Let $\mu$ be a Radon measure on $\mathbb{R}^{d}$ and let $\mathbb{S}$ be the topological support of the measure $\mu$, that is, $\mathbb{S}$ is the minimal closed set whose complement has $\mu$-measure zero. Exotic Brownian motions are diffusion processes associated with Dirichlet forms $\mathcal{E}$ on $L^{2}(\mathbb{S}, \mu)$, where $\mathcal{E}$ is the closure of the form $\mathcal{E}$ (denoted by the same symbol $\mathcal{E}$ ):

$$
\mathcal{E}(f, g)=\int_{\mathbb{S}} \mathbb{D}[f, g] d \mu
$$

with suitably chosen domain $\mathcal{D}_{0}$. Here $\mathbb{D}$ is the canonical square field on $\mathbb{R}^{d}$

$$
\mathbb{D}[f, g]=\frac{1}{2} \sum_{i=1}^{d} \frac{\partial f}{\partial x_{i}} \frac{\partial g}{\partial x_{i}} .
$$

The state space of the diffusion is $\mathbb{S}$ by construction. 
We will take $\mu$ in such a way that it is a countable sum of weighted $d_{n}$-dimensional volume:

$$
\mu=\sum_{n} w_{n} \lambda_{n}
$$

Here $\lambda_{n}$ is the $d_{n}$-dimensional volume on a $d_{n}$-dimensional smooth submanifold $S_{n}$. We assume $1 \leq d_{n} \leq d$. We remark by construction that

$$
\mathbb{S}=\overline{\cup_{n} S_{n}}
$$

Note that dimensions of submanifolds $d_{n}$ may depend on $n$. In the most interesting cases, we assume $d_{n}=d-1$ for all $n$. In such a case $\mu$ is singular to the $d$-dimensional Lebesgue measure; in particular, $\mu$ does not satisfy the doubling condition. When $\mu$ has a well-behaved density with respect to the $d$-dimensional Lebesgue measure, then $(\mathcal{E}, \mathcal{D})$ is called distorted Brownian motion $[2,3]$. We can thus regard exotic Brownian motions as a generalization of distorted Brownian motions.

Under quite mild assumptions it is known that $\left(\mathcal{E}, \mathcal{D}_{0}\right)$ is closable on $L^{2}(\mathbb{S}, \mu)$ and its closure is a strongly local Dirichlet form (see [4]); henceforth the associated diffusion exists, which we call exotic Brownian motion. The purpose of this paper is to prove the parabolic Harnack inequality for (the transition density of) exotic Brownian motions. We will do this in such a way that the dependence on $\mu$, Sobolev and Poincaré constants are clarified.

The diffusion is expected to behave in a rather unusual fashion because of the singularity of $\mu$; for example, in Example 2.1 below, in spite of $\mathbb{S}=\mathbb{R}^{d}(d \geq 2)$ and the diffusion being non-degenerate, the infinitesimal spectral dimension $d_{s}(x)$ at $x \in \mathbb{S}$ - the exponent such that $p(t, x, x) \sim t^{-d_{s}(x) / 2}$ as $t \rightarrow 0$-is different from $d$ in a dense subset of $\mathbb{S}=\mathbb{R}^{d}$. The Harnack inequalities obtained here will be used to show exotic properties of our diffusion processes in a forthcoming paper [9].

\section{Main results and examples}

Let $a=\left(a_{i j}\right)_{i, j=1, \ldots, d}$ be a matrix valued function defined on $\mathbb{S}$ satisfying the following. There exists a positive constant $c_{1}$ such that for $\mu$-a.e. $x$

$$
\begin{aligned}
& c_{1}^{-1}|\xi|^{2} \leq \sum_{i, j=1}^{d} a_{i j}(x) \xi_{i} \xi_{j}, \quad\left|\sum_{i, j=1}^{d} a_{i j}(x) \xi_{i} \eta_{j}\right| \leq c_{1}|\xi||\eta| \\
& \text { for all } \xi=\left(\xi_{i}\right), \eta=\left(\eta_{i}\right) \in \mathbb{R}^{d} .
\end{aligned}
$$


We note that $a$ is not necessary symmetric. Set

$$
\mathcal{E}^{a}(f, g)=\int_{\mathbb{S}} \mathbb{D}^{a}[f, g] d \mu,
$$

where $\mathbb{D}^{a}$ is the square field given by

$$
\mathbb{D}^{a}[f, g]=\frac{1}{2} \sum_{i, j=1}^{d} a_{i j}(x) \frac{\partial f}{\partial x_{i}} \frac{\partial g}{\partial x_{j}} .
$$

When $a$ is the $d$-dimensional unit matrix, we simply write $\mathbb{D}^{a}=\mathbb{D}$ and $\mathcal{E}^{a}=\mathcal{E}$, which coincides with the notation in Section 1. To simplify the notation we often write $\mathbb{D}^{a}[f, f]=\mathbb{D}^{a}[f]$ and $\mathcal{E}^{a}(f, f)=\mathcal{E}^{a}(f)$ and so on. Let $\mathcal{D}_{0}=\left\{\left.f\right|_{\mathbb{S}} ; f \in \mathcal{D}_{0}\left(\mathbb{R}^{d}\right)\right\}$, where $\mathcal{D}_{0}\left(\mathbb{R}^{d}\right)$ is the set defined by

$$
\begin{aligned}
\mathcal{D}_{0}\left(\mathbb{R}^{d}\right)= & \left\{f: \mathbb{R}^{d} \rightarrow \mathbb{R} ; f \text { satisfies }(2.2),(2.3),(2.4)\right\} ; \\
& f \text { is a Lipschitz continuous function with compact support } \\
& \|\mathbb{D}[f, f]\|_{L^{\infty}(\mu)}<\infty \\
& \nabla_{N} f(x)=0 \quad \text { for } \mu \text {-a.e. } x .
\end{aligned}
$$

Here $\nabla_{N}$ is the normal derivative on $S_{k}$, where $\nabla_{N}$ is, by definition, a $\left(d-d_{k}\right)$ dimensional vector perpendicular to $S_{k}$. Recall that each $S_{k}$ is a submanifold, so (2.4) makes sense for $d_{k} \leq d-1$. When $d_{k}=d$, we interpret (2.4) in such a way that (2.4) always holds on $S_{k}$.

We assume that $\mathcal{D}_{0}$ is dense in $C_{0}(\mathbb{S})$ with the uniform norm and also in $L^{2}(\mathbb{S}, \mu)$ and that $\left(\mathcal{E}^{a}, \mathcal{D}_{0}\right)$ is closable on $L^{2}(\mathbb{S}, \mu)$. Then it is clear that its closure $\left(\mathcal{E}^{a}, \mathcal{D}\right)$ is a strongly local regular Dirichlet form; so by using the Dirichlet form theory, one sees there exists a diffusion process associated with $\left(\mathcal{E}^{a}, \mathcal{D}, L^{2}(\mathbb{S}, \mu)\right)$ (see [4]).

A sufficient condition for the above assumption is as follows (see $[\mathbf{8}, \mathbf{9}]$ ).

(1) $S_{m} \cap S_{n}=\emptyset$ if $m \neq n$.

(2) For each $S_{n}$ its closure $\bar{S}_{n}$ is compact and there exists a smooth $d_{n}$-dimensional manifold $\hat{S}_{n}$ such that $\bar{S}_{n} \subset \hat{S}_{n}$.

(3) $\mu\left(\partial S_{n}\right)=0$ for all $n$.

Let $I$ be a compact interval in $\mathbb{R}$. Let $\mathcal{H}^{1}(I \times \mathbb{S})$ denote the closure of $C^{1}(I) \otimes \mathcal{D}_{0}$ with respect to the norm

$$
\sup _{t \in I}\|f(t, \cdot)\|_{L^{2}(\mathbb{S}, \mu)}+\left(\int_{I} \mathcal{E}^{a}(f(t, \cdot), f(t, \cdot)) d t\right)^{1 / 2} .
$$

Note that $t \longmapsto f(t, \cdot) \in L^{2}(\mathbb{S}, \mu)$ is continuous in $t \in I$. 
Let $\mathcal{O}$ be an open set of $\mathbb{S}$. Here $\mathcal{O}$ is endowed with the relative topology from $\mathbb{R}^{d}$. It is clear that $\mu(\mathcal{O})>0$. Set

$$
\begin{gathered}
\mathcal{D}_{0}(\mathcal{O})=\left\{g=\left.f\right|_{\mathcal{O}} ; f \in \mathcal{D}_{0}, g \text { has compact support }\right\}, \\
\mathcal{H}^{1}(I \times \mathcal{O})=\left\{\left.f\right|_{I \times \mathcal{O}} ; f \in \mathcal{H}^{1}(I \times \mathbb{S})\right\} .
\end{gathered}
$$

Set $\mathcal{E}_{\mathcal{O}}^{a}:=\int_{\mathcal{O}} \mathbb{D}^{a} d \mu$ and let $I^{\circ}$ denote the open kernel of $I$. We say a measurable function $u: I \times \mathcal{O} \rightarrow \mathbb{R}$ is a solution in $I \times \mathcal{O}$ of the heat equation associated with the Dirichlet space $\left(\mathcal{E}^{a}, \mathcal{D}, L^{2}(\mathbb{S}, \mu)\right)$ if:

$$
\begin{gathered}
u \in \mathcal{H}^{1}(I \times \mathcal{O}), \\
I^{\circ} \ni t \longmapsto u(t, \cdot) \in L^{2}(\mathcal{O}, \mu) \text { is differentiable, } \\
\int_{I \times \mathcal{O}} \partial_{t} u \varphi d t d \mu+\int_{I} \mathcal{E}_{\mathcal{O}}^{a}(u, \varphi) d t=0,
\end{gathered}
$$

for all $\varphi \in C^{1}(I) \otimes \mathcal{D}_{0}(\mathcal{O})$. For an open interval $J$ we say a function $u$ is a solution in $J \times \mathcal{O}$ if $u$ is a solution in $I \times \mathcal{O}$ for each compact interval $I$ such that $I \subset J$.

Throughout this paper we let $x_{0} \in \mathbb{S}$ be fixed. Let $\rho_{0}$ be a metric on $\mathcal{O}$ that induces the same topology as the original and let $h: \mathbb{R}^{+} \rightarrow \mathbb{R}^{+}$be an increasing function. We set $\rho(x)=h\left(\rho_{0}\left(x_{0}, x\right)\right)$ and $\mathcal{O}(r)=\{x \in \mathbb{S} ; \rho(x)<r\}$. Let

$$
\mu_{r}=\mu(\mathcal{O}(r)) .
$$

Let $r_{0}$ be a positive constant such that $\mathcal{O}\left(r_{0}\right) \subset \mathcal{O}$ and let $\gamma$ be a positive constant such that $2 \gamma<r_{0}$. Assume there exists a positive constant $c_{2}$ such that

$$
c_{2}^{-1} \leq \mathbb{D}[\rho(x)] \leq c_{2} \quad \text { for } \mu \text {-a.e. } x \in \overline{\mathcal{O}(2 \gamma) \backslash} \backslash \mathcal{O}(\gamma / 2) .
$$

Let $\alpha$ be a positive constant such that $1 \leq \alpha<\infty$. For a given $\alpha$ we set

$$
\kappa= \begin{cases}1+2 / \alpha & \text { if } \alpha>2, \\ 1+2 /(\alpha+1) & \text { if } \alpha \leq 2\end{cases}
$$

We assume the following inequalities.

(1) (Sobolev inequality) There exists a positive constant $c_{3}$ such that

$$
\|f\|_{L^{2 \kappa}(\mathcal{O}(r), \mu)}^{2 \kappa} \leq c_{3}\|f\|_{L^{2}(\mathcal{O}(r), \mu)}^{2(\kappa-1)}\left\{\mu_{r}^{1-\kappa}\|f\|_{L^{2}(\mathcal{O}(r), \mu)}^{2}+\|\nabla f\|_{L^{2}(\mathcal{O}(r), \mu)}^{2}\right\}
$$

for all $f \in \mathcal{D}_{0}, \gamma / 2 \leq r \leq \gamma$. 
(2) (Weighted Poincaré inequality) There exists a positive constant $c_{4}$ such that

$$
\|f-\bar{f}\|_{L^{2}\left(\mathcal{O}(2 \gamma), \psi^{2} \mu\right)}^{2} \leq c_{4} \gamma^{2}\|\nabla f\|_{L^{2}\left(\mathcal{O}(2 \gamma), \psi^{2} \mu\right)}^{2} .
$$

Here $\bar{f}=\left\{\int_{\mathcal{O}(2 \gamma)} \psi^{2} d \mu\right\}^{-1} \int_{\mathcal{O}(2 \gamma)} f \psi^{2} d \mu$ and $\psi(x)=\bar{\psi}(\rho(x))$, where $\bar{\psi}: \mathbb{R}^{+} \rightarrow \mathbb{R}^{+}$is the function defined by $\bar{\psi}(t)=1$ for $0 \leq t \leq \gamma$, $\bar{\psi}(t)=-\gamma^{-1} t+2$ for $\gamma \leq t \leq 2 \gamma$ and $\bar{\psi}(t)=0$ for $2 \gamma \leq t<\infty$.

THEOREM 2.1. Let $I_{\mp}$ be closed intervals such that $I_{-}=\gamma^{2}[-3 / 4,-1 / 4]+a$ and $I_{+}=\gamma^{2}[1 / 4,1]+$ a for some $a \in \mathbb{R}$. Let $I$ be an open interval such that $\gamma^{2}[-1,1]+a \subset I$. Let $u$ be a non-negative solution in $I \times \mathcal{O}$. Let $\mathcal{O}(\gamma / 2)$ be as above. Then there exists a version of $u(t, x)$ such that

$$
\sup _{I_{-} \times \mathcal{O}(\gamma / 2)} u(t, x) \leq c_{5} \inf _{I_{+} \times \mathcal{O}(\gamma / 2)} u(t, x),
$$

where $c_{5}$ is a constant depending only on $c_{1}, c_{2}, c_{3}, c_{4}, \alpha$ and

$$
\gamma^{-2 /(\kappa-1)} \mu_{\gamma} \text {. }
$$

Remark 2.1. (1) More precisely, the constant $c_{5}$ is given by $c_{5}=\exp \left\{2 c_{6} c_{7}\right\}$, where $c_{6}$ and $c_{7}$ are constants given by

$$
\begin{aligned}
& c_{6}=\left(\frac{2}{\gamma}\right)^{\bar{\kappa} \tau(\sigma-1)} \cdot\left\{\sum_{j=0}^{\infty}\left(\frac{1+\tau}{\sigma \tau}\right)^{j}((1+j)(2+j))^{\bar{\kappa} \tau(\sigma-1)}\right\}, \\
& c_{7}=\max \left\{\sigma c_{8}\left(\frac{\gamma}{2}\right)^{\bar{\kappa} \tau(\sigma-1)}, 2 c_{1} c_{4}\left(2 c_{9} \gamma^{2-\bar{\kappa}} \mu_{\gamma}\right)^{\tau(\sigma-1)}\right\} .
\end{aligned}
$$

Here the constant $\bar{\kappa}$ is defined by

$$
\bar{\kappa}=\frac{\kappa}{\kappa-1}= \begin{cases}\frac{\alpha+2}{2} & (2<\alpha), \\ \frac{\alpha+3}{2} & (1 \leq \alpha \leq 2),\end{cases}
$$

and $c_{8}=\max \{x \in A\}$ if $A \neq \emptyset$ and $c_{8}=0$ if otherwise, where

$$
A=\left\{x \in \mathbb{R}^{+} ; \frac{1}{2 c_{1}}=\frac{1}{x(\sigma-1)} \log \left(\frac{x}{2 c_{1} c_{4}}\right)\right\} .
$$

$\sigma$ and $\tau$ are any numbers satisfying $1<\sigma, \tau<\infty,(1+\tau) /(\sigma \tau)<1$ and $c_{9}$ is a positive constant defined by

$$
\begin{aligned}
c_{9} & =c_{10} \cdot\left(c_{3} c_{1}\right)^{1 /(\kappa-1)} \cdot\left(\max \left\{1, \gamma^{2} \mu_{\gamma}^{1-\kappa} / c_{1}\right\}\right)^{1 /(\kappa-1)} \\
& =c_{10} \cdot\left(c_{3} \max \left\{c_{1}, \gamma^{2} \mu_{\gamma}^{1-\kappa}\right\}\right)^{1 /(\kappa-1)},
\end{aligned}
$$

where $c_{10}$ is a positive constant depending only on $\left(\kappa, c_{1}, c_{2}\right)($ see $(6.16))$. 
(2) A simple calculation shows that, for given $c_{1}, c_{2}, c_{3}, c_{4}$ and $\alpha$, we can take a non-negative and non-decreasing function $C:(0, \infty) \rightarrow(0, \infty)$ such that $\lim _{s \rightarrow \infty} C(s)=\infty, \lim _{s \rightarrow 0} C(s)>0$ and that

$$
c_{5}=C\left(\gamma^{-2 /(\kappa-1)} \mu_{\gamma}\right) \text {. }
$$

(3) Let $x_{0}$ denote the center of domains. Suppose $\alpha>d_{\mathrm{H}}>d-1$, where $d_{\mathrm{H}}$ is the Hausdorff dimension of $\mathbb{S}$, and (2.11) and (2.12) hold for $\gamma_{k}=C^{-k}(C>1$, $k=1,2, \ldots$ ) with constants uniform in $k$. Suppose $d_{n}=d-1$ for all $n$ and the doubling condition is not satisfied. This is the situation in Examples 2.1 and 2.2. If $x_{0} \in \cup_{n} S_{n}$, then $\mu_{\gamma_{k}} \sim \gamma_{k}^{-(d-1)}$ as $\gamma_{k} \rightarrow 0$; so $\sup _{k} c_{5}\left(\gamma_{k}\right)=\infty$. We remark that, in the present situation, one cannot expect the global Harnack inequality, which means (2.13) holds with some $c_{5}\left(\gamma_{k}\right)>0$ such that

$$
\sup _{k \in \mathbb{N}, x_{0} \in \mathbb{S}} c_{5}\left(\gamma_{k}\right)<\infty .
$$

This is because $\sup _{k \in \mathbb{N}, x_{0} \in \mathbb{S}} c_{5}\left(\gamma_{k}\right)<\infty$, together with (2.11) and (2.12) for $\gamma(k)=3^{-k}$, implies the doubling condition of $\mu$ by the argument of Grigor'yan [5]. The doubling condition is commonly assumed in $[\mathbf{5}, \mathbf{1 0}, \mathbf{1 1}]$. We emphasize that, even if $\mu$ does not satisfy the doubling condition, one can prove (non-uniform) Harnack inequalities in some cases. Such a Harnack inequality is weaker than those in $[5, \mathbf{1 0}, \mathbf{1 1}]$. It is still useful to obtain the locally uniform lower bound of transition densities of associated diffusion processes, as this yields the non-degeneracy of diffusion processes.

We now show examples.

Example 2.1. Exotic Brownian motions over $\mathbb{R}^{d}(d \geq 2)$. Let $d \geq 2$ and let $N$ be a natural number such that $N \geq 2$. Let $\mathrm{B}=\cup_{i=1}^{d}\left\{x=\left(x_{1}, \ldots, x_{d}\right) \in \mathbb{R}^{d} ; x_{i} \in \mathbb{Z}, x_{j} \notin\right.$ $\mathbb{Z}$ for all $j \neq i$ \} and $\mathrm{B}^{n}=(1 / N)^{n} \mathrm{~B}$. We set

$$
S_{n}=\mathrm{B}^{n+1} \backslash \mathrm{B}^{n} .
$$

For given $\alpha>d$ we take the weight $w_{n}$ in (1.2) such that

$$
w_{n}=N^{-(\alpha-d+1) n} .
$$

Then $\mu=\mu^{[\alpha]}$ is a Radon measure on $\mathbb{R}^{d}$ whose support equals $\mathbb{R}^{d}$. The measure $\mu$ satisfies the self-similarity in the sense that

$$
\mu(N A)=N^{\alpha} \cdot \mu(A) \quad \text { for all } A \in \mathfrak{B}\left(\mathbb{R}^{d}\right)
$$


and the cell translation invariance in the sense of [8]. By using the method developed in [8] we see that $p$ satisfies a global Gaussian upper bound; there exists a positive constant $c_{11}$ (and a version $p$ ) such that

$$
p(t, x, y) \leq c_{11} t^{-\alpha / 2} e^{-|x-y|^{2} / c_{11} t} \quad \text { for all } 0<t<\infty, x, y \in \mathbb{R}^{d}
$$

and that geometric inequalities (2.11) and (2.12) hold for sufficiently many domains. From this and Theorem 2.1, one can prove the transition densities $p=p(t, x, y)$ are locally uniformly positive in the sense of (2.19) below-henceforth, diffusions are non-degenerate. In addition, there exists a dense subset $R$ in $\mathbb{R}^{d}$ satisfying the following. The transition densities are continuous in $(0, \infty) \times R \times R$ and

$$
c_{12}^{-1} t^{-\alpha / 2} \leq p(t, x, x) \leq c_{12} t^{-\alpha / 2} \text { for all } 0<t<1, x \in R .
$$

Here $c_{12}=c_{12}(x)$ are positive constants depending on $x \in R$. The last inequality means that the short time asymptotic behavior of the transition density is different from the standard Brownian motion in the dense subset $R$, in spite of the diffusion begin non-degenerate and moving around over $\mathbb{R}^{d}$.

Example 2.2. Exotic Brownian motions on Sierpinski carpets [8]. Let $F$ denote the $d$-dimensional Sierpinski carpet and $d_{\mathrm{H}}$ the Hausdorff dimension of $F$. If we replace $N$ by $3, \mathbb{R}^{d}$ by $F$ and $d$ by $d_{\mathrm{H}}$ in Example 2.1, then the same conclusion as Example 2.1 holds.

Example 2.3. Exotic Brownian motions on bubbles. Let $d \geq 2$ and let $B_{n}$ denote a closed ball with radius $r_{n}$. Let $S_{n}$ denote the boundary of $B_{n}$. Assume

$$
\begin{aligned}
& B_{n} \cap B_{m}=\emptyset \quad \text { for } n \neq m \\
& \overline{\cup_{n \in \mathbb{N}} B_{n}}=\mathbb{R}^{d} .
\end{aligned}
$$

Let us take the weight $w_{n}$ in such a way that $w_{n}=r_{n}$, then it is known that a global Gaussian upper bound holds; there exists a positive constant $c_{13}$ such that

$$
p(t, x, y) \leq c_{13} t^{-d / 2} e^{-|x-y|^{2} / c_{13} t} \text { for a.e. } 0<t<\infty, x, y, \in \mathbb{S} .
$$

Here and below a.e. is with respect to $d t \times \mu \times \mu$. In addition, for sufficiently many domains (2.11) and (2.12), henceforth (2.13) by Theorem 2.1, are satisfied with $\alpha=d$. From this and (2.18) above we see that $p$ is locally uniform positive in the sense that for any compact set $K \subset(0, \infty) \times \mathbb{S} \times \mathbb{S}$ there exists a positive constant $c_{14}=c_{14}(K)$ such that

$$
0<c_{14} \leq p(t, x, y) \quad \text { for a.e. }(t, x, y) \in K \text {. }
$$


There is a celebrated work by Moser [7], which together with [6] proves parabolic Harnack inequalities for uniformly elliptic operators. Since then, many extensions have been made (see, for example, $[\mathbf{1 0 , 1 1}]$ and references therein). In these works the doubling condition is a pivotal assumption. One of our contributions is that we carry out Moser's argument without the doubling condition. This is imperative, because the examples cited above do not satisfy the doubling condition. The diffusions in these examples are somehow exotic, but still well-behaved, diffusions that have various invariance. To apply Moser's theory to these diffusions we relax the assumptions. Our second contribution is to clarify the dependence of $c_{5}$ on the constants such as $c_{1}$, $c_{3}$, etc. This refinement will also be used in [9] to obtain fine estimates of diffusions on $\mathbb{R}^{d}$.

As for work on Moser's iterations on fractal sets, we refer to the work of Barlow and Bass [1]. In their paper the reference measure essentially satisfies the doubling condition.

The organization of the rest of this paper is as follows. In Section 3 we prepare three lemmas, Lemmas 3.1-3.3. In Section 4 we prove the main theorem by using the lemmas in Section 3. In Section 5 we do some calculations on Dirichlet form. In Sections 6-8, we prove Lemmas 3.1-3.3, respectively.

\section{Preliminary lemmas}

In this section we prepare three lemmas which we will prove in Sections $6-8$. In the next section we will prove the main theorem by using these lemmas.

Let $J_{-}(r)=\left[-r^{2}, 0\right], J_{+}(r)=\left[0, r^{2}\right]$ and $J(r)=J_{-}(r) \cup J_{+}(r)$. Set $R_{ \pm}(r)=$ $J_{ \pm}(r) \times \mathcal{O}(r)$ and $R(r)=J(r) \times \mathcal{O}(r)$. Let $\bar{\mu}$ denote the product measure $\bar{\mu}=d t \times \mu$ and let $\kappa$ and $\bar{\kappa}$ be as in Section 2. We recall

$$
\bar{\kappa}= \begin{cases}\frac{\alpha+2}{2} & (2<\alpha), \\ \frac{\alpha+3}{2} & (1 \leq \alpha \leq 2) .\end{cases}
$$

LEMMA 3.1. Let $u$ be a uniformly positive solution in a neighborhood of $R(\gamma)$; then for all $\gamma / 2<r_{1}<r_{2} \leq \gamma$ and $0<p<1 /\left(2 c_{1}\right)$ we have

$$
\begin{aligned}
\sup _{R\left(r_{1}\right)} u & \leq\left\{\frac{c_{9}}{\left(r_{2}-r_{1}\right)^{2 \bar{\kappa}}}\right\}^{1 / p}\|u\|_{L^{p}\left(R\left(r_{2}\right), \bar{\mu}\right),} \\
\sup _{R_{-}\left(r_{1}\right)} \frac{1}{u} & \leq\left\{\frac{c_{9}}{\left(r_{2}-r_{1}\right)^{2 \bar{\kappa}}}\right\}^{1 / p}\left\|\frac{1}{u}\right\|_{L^{p}\left(R_{-}\left(r_{2}\right), \bar{\mu}\right)} .
\end{aligned}
$$


LEMMA 3.2. Let $u$ be a uniformly positive solution in a neighborhood of $R(2 \gamma)$. Let $c_{15}$ be the constant defined by

$$
c_{15}=2 c_{1}^{3} c_{2} \frac{\mu_{2 \gamma}}{\mu_{\gamma}}-\int_{\mathcal{O}(\gamma)} \log u(0, x) d \mu .
$$

Then for all $s>0$ the following holds:

$$
\begin{gathered}
\bar{\mu}\left(R_{-}(\gamma) \cap\left\{u>e^{s-c_{15}}\right\}\right) \leq \frac{2 c_{1} c_{4} \gamma^{2} \mu_{\gamma}}{s} ; \\
\bar{\mu}\left(R_{+}(\gamma) \cap\left\{u<e^{-s-c_{15}}\right\}\right) \leq \frac{2 c_{1} c_{4} \gamma^{2} \mu_{\gamma}}{s} .
\end{gathered}
$$

Let $\{Q(r)\}(\gamma / 2 \leq r \leq \gamma)$ denote any family of measurable subsets in $\mathbb{R}^{d+1}$ such that $Q\left(r_{1}\right) \subset Q\left(r_{2}\right)$ for $r_{1} \leq r_{2}$.

LEMMA 3.3. Let $\beta$ and $\theta$ be constants such that $0<\beta<\infty$ and $1 / 2 \leq \theta<1$. Let $f$ be a positive measurable function satisfying the following. For positive constants $c_{16}$ and $c_{17}$

$$
\begin{gathered}
\sup _{Q\left(r_{1}\right)} f \leq\left\{\frac{c_{16}}{\left(r_{2}-r_{1}\right)^{\beta} \bar{\mu}(Q(\gamma))}\right\}^{1 / p}\|f\|_{L^{p}\left(Q\left(r_{2}\right), \bar{\mu}\right)} \\
\text { for all } \theta \gamma \leq r_{1}<r_{2} \leq \gamma, 0<p<\frac{1}{2 c_{1}} \\
\bar{\mu}\left(f \geq e^{s}\right) \leq \frac{2 c_{1} c_{17}}{s} \cdot \bar{\mu}(Q(\gamma)),
\end{gathered}
$$

then

$$
\sup _{Q(\theta \gamma)} f \leq c_{18}
$$

where $c_{18}=c_{18}\left(\gamma, \theta, \beta, c_{16}, c_{17}\right)$ is given by

$$
c_{18}=\exp \left\{c_{19} c_{20}\right\} .
$$

Here $c_{19}=c_{19}(\gamma, \theta, \beta, \sigma, \tau)$ and $c_{20}=c_{20}\left(\gamma, \theta, \beta, c_{16}, c_{17}, \sigma, \tau\right)$ are constants given by

$$
\begin{aligned}
& c_{19}=\left(\frac{1}{\gamma(1-\theta)}\right)^{\beta \tau(\sigma-1)} \sum_{j=0}^{\infty}\left(\frac{1+\tau}{\sigma \tau}\right)^{j}((1+j)(2+j))^{\beta \tau(\sigma-1)}, \\
& c_{20}=\max \left\{\sigma c_{21}(\gamma(1-\theta))^{\beta \tau(\sigma-1)}, 2 c_{1} c_{17}\left(2 c_{16}\right)^{\tau(\sigma-1)}\right\}
\end{aligned}
$$

where $c_{21}$ is defined similarly to $c_{8}$ with the replacement of $c_{4}$ by $c_{17}$ and $\sigma, \tau$ are positive numbers such that $((1+\tau) /(\sigma \tau))<1$. 


\section{Proof of the main theorem}

In this section we deduce Theorem 2.1 from the lemmas in Section 3.

LEMMA 4.1. Let $u$ be a uniformly positive solution in a neighborhood of $R(2 \gamma)$. Then

$$
\sup _{R_{-}(\gamma / 2)} u \leq c_{18} e^{-c_{15}}
$$

Here $c_{18}$ is given by (3.9) with the choice of $\theta=1 / 2, \beta=\bar{\kappa}, c_{16}=c_{9} \gamma^{2} \mu_{\gamma} / \gamma^{\bar{\kappa}}$ and $c_{17}=c_{4}$.

Proof. Apply Lemma 3.3 to $f=u e^{c_{15}}, Q(r)=\left[-r^{2}-\gamma^{2} / 2, r^{2}-\gamma^{2} / 2\right] \times \mathcal{O}(r), \theta, \beta$, $c_{16}$ and $c_{17}$ as above. Since $Q(r)$ thus defined are translations of $R(r)$ in time, one can apply Lemma 3.1 to obtain (3.1) for $Q(r)$; so we obtain (3.6). Since $Q(\gamma)=R_{-}(\gamma)$, we obtain (3.7) by (3.4). Hence the assumptions of Lemma 3.3 are fulfilled, which yields (4.1).

LEMMA 4.2. Let $u$ be a uniformly positive solution in a neighborhood of $R(2 \gamma)$. Let $R_{*}(r)=\left[\gamma^{2}-r^{2}, \gamma^{2}\right] \times \mathcal{O}(r)$. Then

$$
\sup _{R_{*}(\gamma / 2)} u^{-1} \leq c_{18} e^{c_{15}} .
$$

Here $c_{18}$ is given by (3.9) with the choice of $\theta=1 / 2, \beta=\bar{\kappa}, c_{16}=c_{9} \gamma^{2} \mu_{\gamma} / \gamma^{\bar{\kappa}}$ and $c_{17}=c_{4}$.

Proof. Note that $R_{*}(r)$ is the translation of $R_{-}(r)$ in time. Apply Lemma 3.3 to $f=\left(u e^{c_{15}}\right)^{-1}, Q(r)=R_{*}(r), \theta=1 / 2$, we obtain (4.2) from Lemma 3.1, (3.2), (3.5) and Lemma 3.3.

Proof of Theorem 2.1. Without loss of generality, one can assume $a=0$. Then we see $I_{-} \times \mathcal{O}(\gamma / 2)=R_{-}(\gamma / 2)$ and $I_{+} \times \mathcal{O}(\gamma / 2)=R_{*}(\gamma / 2)$.

Let $u_{\epsilon}=u+\epsilon$, where $\epsilon$ is an arbitrary positive constant, then $u_{\epsilon}$ is a uniformly positive solution. So we can apply Lemmas 4.1 and 4.2 to $u_{\epsilon}$. Multiplying (4.1) and (4.2) we obtain (2.13) for $u_{\epsilon}$. Since the constant $c_{5}$ is independent of $\epsilon,(2.13)$ is also valid for $u$. This completes the proof.

\section{Calculation based on Dirichlet forms}

In this section we prove an equality for solutions of (2.5)-(2.7) by using the Dirichlet form structure. We will use this equality in the subsequent sections to deduce basic inequalities. 
Let $u$ be a solution of (2.5)-(2.7) in $I \times \mathcal{O}$. We assume $u$ is positive. In the subsequent sections we need inequalities for functions $v$ of the form

$$
v:=g(u)
$$

Here $g:(0, \infty) \rightarrow \mathbb{R}$ is a function. We will take $g(t)=t^{p / 2}$ in Lemma 6.1 and $g(t)=-\log t$ in Lemma 7.1 .

Lemma 5.1. Let $I=[a, b], \phi \in C^{1}(I) \otimes \mathcal{D}_{0}(\mathcal{O})$ and let $f, g:(0, \infty) \rightarrow \mathbb{R}$ be smooth functions. Set $U=f(u(t, x)), V=g(u(t, x))$ and let $\eta= \pm 1$ be a constant. Suppose $f^{\prime \prime}=\eta\left(g^{\prime}\right)^{2}$. Then

$$
\begin{aligned}
\left.\int_{\mathcal{O}} U(t, \cdot) \phi^{2} d \mu\right|_{a} ^{b}-2 \int_{I \times \mathcal{O}} U \partial_{t} \phi \phi d \bar{\mu} \\
\\
\quad+\int_{I}\left\{\eta \mathcal{E}_{\mathcal{O}}^{a}\left(V, V ; \phi^{2}\right)+2 \mathcal{E}_{\mathcal{O}}^{a}(U, \phi ; \phi)\right\} d t=0 .
\end{aligned}
$$

Here $\mathcal{E}_{\mathcal{O}}^{a}(\cdot, * ; h)=\int_{\mathcal{O}} \mathbb{D}^{a}[\cdot, *] h d \mu$ and $\bar{\mu}=d t \times \mu$ as before.

Proof. Let $\varphi:=f^{\prime}(u) \phi^{2}$, then the direct calculation shows

$$
\begin{aligned}
\mathbb{D}^{a}[u, \varphi] & =\mathbb{D}^{a}[u, u] f^{\prime \prime}(u) \phi^{2}+2 \mathbb{D}^{a}[u, \phi] f^{\prime}(u) \phi \\
& =\eta \mathbb{D}^{a}[g(u), g(u)] \phi^{2}+2 \mathbb{D}^{a}[f(u), \phi] \phi .
\end{aligned}
$$

Hence (5.1) follows immediately from this and (2.7) with integration by parts in $t$.

\section{Proof of Lemma 3.1}

Let $J^{*}(r)$ be one of $J(r), J_{ \pm}(r)$, which were defined at the beginning of Section 3 . Let $R^{*}(r)=J^{*}(r) \times \mathcal{O}(r)$.

LEMMA 6.1. Let $v=u^{p / 2}, \epsilon(p)=\frac{1}{2}\left|1-p^{-1}\right|$, let $\gamma / 2 \leq r_{1}<r_{2} \leq \gamma$ and let $c_{22}=c_{22}(p)$ be the constant defined by

$$
c_{22}(p)=2+\frac{c_{1}^{3} c_{2}}{\epsilon(p)} .
$$

We consider inequalities for domains of the form $R^{*}(r)=J^{*}(r) \times \mathcal{O}(r)$

$$
\sup _{t \in J^{*}\left(r_{1}\right)}\|v(t, \cdot)\|_{L^{2}\left(\mathcal{O}\left(r_{1}\right), \mu\right)}^{2}+\frac{2 \epsilon(p)}{c_{1}}\|\nabla v\|_{L^{2}\left(R^{*}\left(r_{1}\right), \bar{\mu}\right)}^{2} \leq \frac{c_{22}(p)}{\left(r_{2}-r_{1}\right)^{2}}\|v\|_{L^{2}\left(R^{*}\left(r_{2}\right), \bar{\mu}\right)}^{2} .
$$


(1) Let $0<p<1$; then (6.2) holds for $J^{*}=J_{+}$and $R^{*}=R_{+}$.

(2) Let $1<p<\infty$; then (6.2) holds for $J^{*}=J_{-}$and $R^{*}=R_{-}$.

(3) Let $0<p<\infty$ and $p \neq 1$; then (6.2) holds for $J^{*}=J$ and $R^{*}=R$.

Proof. We prove (1), so assume $0<p<1$. Let us take $f(t)=1 / 4 t^{p}, g(t)=$ $\sqrt{2 \epsilon(p)} t^{p / 2}$ and $\eta=-1$ in Lemma 5.1; then $f^{\prime \prime}=\eta\left(g^{\prime}\right)^{2}$ is satisfied. So we can apply Lemma 5.1. Let $\phi$ be such that $\phi\left(r_{2}, x\right)=0$. Let $I_{r}=\left[r, r_{2}\right]$ for $r_{1} \leq r<r_{2}$ and $I=\left[r_{1}, r_{2}\right]$. Then by $(5.1)$ and the relation

$$
V^{2}=8 \epsilon(p) U
$$

which follows from $g^{2}=8 \epsilon(p) f, V=g(u)$ and $U=f(u)$, yields

$$
\begin{array}{rl}
\frac{1}{8 \epsilon(p)} \int_{\mathcal{O}} V^{2}\left(r_{1}, \cdot\right) \phi^{2} & d \mu+\int_{I_{r}} \mathcal{E}_{\mathcal{O}}^{a}\left(V, V ; \phi^{2}\right) d t \\
= & \frac{-1}{4 \epsilon(p)} \int_{I_{r} \times \mathcal{O}} V^{2} \partial_{t} \phi \phi d \bar{\mu}+\frac{1}{2 \epsilon(p)} \int_{I_{r}} \mathcal{E}_{\mathcal{O}}^{a}(V, \phi ; V \phi) d t
\end{array}
$$

Since

$$
\frac{1}{2 \epsilon(p)} \mathcal{E}_{\mathcal{O}}^{a}(V, \phi ; V \phi) \leq \frac{1}{2} \mathcal{E}_{\mathcal{O}}^{a}\left(V, V ; \phi^{2}\right)+\frac{c_{1}^{2}}{8 \epsilon(p)^{2}} \mathcal{E}_{\mathcal{O}}^{a}\left(\phi, \phi ; V^{2}\right),
$$

we obtain

$$
\begin{array}{rl}
\frac{1}{8 \epsilon(p)} \int_{\mathcal{O}} V^{2}\left(r_{1}, \cdot\right) \phi^{2} & d \bar{\mu}+\frac{1}{2} \int_{I_{r}} \mathcal{E}_{\mathcal{O}}^{a}\left(V, V ; \phi^{2}\right) d t \\
\leq & \frac{-1}{4 \epsilon(p)} \int_{I_{r} \times \mathcal{O}} V^{2} \partial_{t} \phi \phi d \bar{\mu}+\frac{c_{1}^{2}}{8 \epsilon(p)^{2}} \int_{I_{r}} \mathcal{E}_{\mathcal{O}}^{a}\left(\phi, \phi ; V^{2}\right) d t
\end{array}
$$

Taking $\phi$ in such a way that $\phi(t, x)=1$ for $(t, x) \in R_{+}\left(r_{1}\right), \phi(t, x)=0$ for $(t, x) \notin R_{+}\left(r_{2}\right),\left|\mathbb{D}^{a}[\phi, \phi]\right| \leq c_{1} c_{2} /\left(r_{2}-r_{1}\right)^{2}$ and $\left|\partial_{t} \phi\right| \leq 1 /\left(r_{2}-r_{1}\right)^{2}$ yields (1). The proof of (2) is similar and (3) follows immediately from (1) and (2).

LEMMA 6.2. Let $c_{23}(p)$ and $c_{24}(p)$ be positive constants defined by

$$
\begin{aligned}
& c_{23}(p)=2 c_{1}\left(\frac{\gamma^{2} \mu_{\gamma}^{1-\kappa}}{c_{1}}+\frac{1}{4 \epsilon(p)}\right), \\
& c_{24}(p)=\left\{c_{3} c_{23}(p)\right\}^{1 / \kappa p} c_{22}(p)^{1 / p} .
\end{aligned}
$$

Then for each $\gamma / 2 \leq r_{1}<r_{2} \leq \gamma$ and $0<p<\infty, p \neq 1$ we have

$$
\|u\|_{L^{\kappa p}\left(R\left(r_{1}\right), \bar{\mu}\right)} \leq \frac{c_{24}(p)}{\left(r_{2}-r_{1}\right)^{2 / p}}\|u\|_{L^{p}\left(R\left(r_{2}\right), \bar{\mu}\right)} .
$$


Proof. Applying (2.11) to $v=u^{p / 2}$ and integrating over $\left[-r_{1}^{2}, r_{1}^{2}\right]$ in $t$ we obtain

$$
\begin{aligned}
\|v\|_{L^{2 \kappa}\left(R\left(r_{1}\right), \bar{\mu}\right)}^{2 \kappa} \leq c_{3}\left\{\sup _{t}\|v(t, \cdot)\|_{L^{2}\left(\mathcal{O}\left(r_{1}\right), \mu\right)}^{2(\kappa-1)}\right\} & \cdot\left\{\mu_{\gamma}^{1-\kappa}\|v\|_{L^{2}\left(R\left(r_{1}\right), \bar{\mu}\right)}^{2}+\|\nabla v\|_{L^{2}\left(R\left(r_{1}\right), \bar{\mu}\right)}^{2}\right\}
\end{aligned}
$$

By (3) of Lemma 6.1 we have

$$
\sup _{t}\|v(t, \cdot)\|_{L^{2}\left(\mathcal{O}\left(r_{1}\right), \mu\right)}^{2(\kappa-1)} \leq\left(\frac{c_{22}(p)}{\left(r_{2}-r_{1}\right)^{2}}\right)^{\kappa-1}\|v\|_{L^{2}\left(R\left(r_{2}\right), \bar{\mu}\right)}^{2(\kappa-1)} .
$$

By (3) of Lemma 6.1 and $\left|J\left(r_{1}\right)\right| \leq 2 \gamma^{2}$ we have

$$
\frac{1}{2 \gamma^{2}}\|v\|_{L^{2}\left(R\left(r_{1}\right), \bar{\mu}\right)}^{2}+\frac{2 \epsilon(p)}{c_{1}}\|\nabla v\|_{L^{2}\left(R\left(r_{1}\right), \bar{\mu}\right)}^{2} \leq \frac{c_{22}(p)}{\left(r_{2}-r_{1}\right)^{2}}\|v\|_{L^{2}\left(R\left(r_{2}\right), \bar{\mu}\right)}^{2},
$$

which immediately implies

$$
\mu_{\gamma}^{1-\kappa}\|v\|_{L^{2}\left(R\left(r_{1}\right), \bar{\mu}\right)}^{2}+\|\nabla v\|_{L^{2}\left(R\left(r_{1}\right), \bar{\mu}\right)}^{2} \leq c_{23}(p)\left(\frac{c_{22}(p)}{\left(r_{2}-r_{1}\right)^{2}}\right)\|v\|_{L^{2}\left(R\left(r_{2}\right), \bar{\mu}\right)}^{2} .
$$

Putting this and (6.7) into (6.6) yields

$$
\|v\|_{L^{2 \kappa}\left(R\left(r_{1}\right), \bar{\mu}\right)}^{2 \kappa} \leq c_{3} c_{23}(p) \cdot\left(\frac{c_{22}(p)}{\left(r_{2}-r_{1}\right)^{2}}\right)^{\kappa}\|v\|_{L^{2}\left(R\left(r_{2}\right), \bar{\mu}\right)}^{2 \kappa} .
$$

Recalling $v=u^{p / 2}$ and $R\left(r_{1}\right) \subset R\left(r_{2}\right)$ we obtain (6.5).

Proof of Lemma 3.1. For $m \in \mathbb{N}=\{0,1,2, \ldots\}$ let

$$
\begin{aligned}
r_{1, m} & =r_{1}+\left(r_{2}-r_{1}\right) 2^{-(m+1)} \\
r_{2, m} & =r_{1}+\left(r_{2}-r_{1}\right) 2^{-m} \\
p_{m} & =p \kappa^{m},
\end{aligned}
$$

where $p=\frac{1}{2} \kappa^{n}(\kappa+1)$ for some $n \in \mathbb{Z}$. We choose $n$ in such a way that

$$
\begin{aligned}
&\left|p_{m}-1\right| \geq 1-\kappa^{-1} \quad \text { for all } m \in \mathbb{N}, \\
& \frac{1}{\epsilon\left(p_{m}\right)} \leq \frac{\kappa^{m}}{1-\kappa^{-1}} \quad \text { for all } m \in \mathbb{N} .
\end{aligned}
$$

We note by $p_{m}=p \kappa^{m}$,

$$
\begin{gathered}
\sum_{m=0}^{\infty} \frac{1}{p_{m}}=\frac{\kappa}{p(\kappa-1)}=\frac{\bar{\kappa}}{p}, \\
\sum_{m=0}^{\infty} \frac{m+1}{p_{m}}=\frac{\kappa^{2}}{p(\kappa-1)^{2}}=\frac{\bar{\kappa}^{2}}{p} .
\end{gathered}
$$


By (6.5), $r_{1, \infty}=r_{2, \infty}=r_{1}$ and $r_{2,0}=r_{2}$ we have

$$
\begin{aligned}
\sup _{R\left(r_{1}\right)} u & =\lim _{m \rightarrow \infty}\|u\|_{L^{p m}\left(R\left(r_{1}(m)\right), \bar{\mu}\right)} \\
& \leq\left\{\prod_{m \in \mathbb{N}} c_{24}\left(p_{m}\right) \frac{2^{(m+1) / p_{m}}}{\left(r_{2}-r_{1}\right)^{2 / p_{m}}}\right\}\|u\|_{L^{p}\left(R\left(r_{2}\right), \bar{\mu}\right)} \\
& =\left\{\prod_{m \in \mathbb{N}} c_{24}\left(p_{m}\right)\right\} \cdot\left(\frac{2^{\bar{\kappa}^{2}}}{\left(r_{2}-r_{1}\right)^{2 \bar{\kappa}}}\right)^{1 / p}\|u\|_{L^{p}\left(R\left(r_{2}\right), \bar{\mu}\right)}
\end{aligned}
$$

Here we used (6.10) and (6.11) for the last line. Let $c_{25}=\prod_{m \in \mathbb{N}}\left\{c_{3} c_{23}\left(p_{m}\right)\right\}^{1 / \kappa p_{m}}$ and $c_{26}=\prod_{m \in \mathbb{N}} c_{22}\left(p_{m}\right)^{1 / p_{m}}$, then by definition we have

$$
\prod_{m \in \mathbb{N}} c_{24}\left(p_{m}\right)=c_{25} \cdot c_{26}
$$

By (6.9) and (6.11) and the definition of $c_{23}$ and $c_{22}$, we easily see there exist positive constants $c_{27}$ and $c_{28}$ depending only on $\kappa$ and $\left(\kappa, c_{1}, c_{2}\right)$, respectively, satisfying the following:

$$
\begin{aligned}
c_{25} & \leq \prod_{m \in \mathbb{N}}\left\{2 c_{3} c_{1}\left(\frac{\gamma^{2} \mu_{\gamma}^{1-\kappa}}{c_{1}}+\frac{\kappa^{m+1}}{\kappa-1}\right)\right\}^{1 / \kappa p_{m}} \\
& \leq\left[c_{27}\left(c_{3} c_{1}\right)^{1 /(\kappa-1)}\left\{\max \left\{\frac{\gamma^{2} \mu_{\gamma}^{1-\kappa}}{c_{1}}, 1\right\}\right\}^{1 /(\kappa-1)}\right]^{1 / p}, \\
c_{26} & \leq \prod_{m \in \mathbb{N}}\left\{2+c_{1}^{3} c_{2}\left(\frac{\kappa^{m+1}}{\kappa-1}\right)\right\}^{1 / p_{m}}=c_{28}^{1 / p} .
\end{aligned}
$$

Putting (6.13), (6.14) and (6.15) into (6.12) and letting

$$
c_{10}=c_{27} c_{28} 2^{\bar{\kappa}^{2}}
$$

proves (3.1). The inequality (3.2) is derived similarly to (2) of Lemma 6.1.

\section{Proof of Lemma 3.2}

LEMMA 7.1. Let $v=-\log u$ and $\psi \in \mathcal{D}_{0}(\mathcal{O})$, then

$$
\left.\int v \psi^{2} d \mu\right|_{t_{1}} ^{t_{2}}+\frac{1}{2} \int_{t_{1}}^{t_{2}} \mathcal{E}_{\mathcal{O}}^{a}\left(v, v ; \psi^{2}\right) d t \leq 2 c_{1}^{2} \mathcal{E}_{\mathcal{O}}^{a}(\psi, \psi)\left(t_{2}-t_{1}\right) .
$$


Proof. Let us take in Lemma 5.1 as $f(t)=-\log t, g(t)=\log t, I=\left[t_{1}, t_{2}\right]$, $\phi=1 \otimes \psi$ and $\eta=1$. Then $U=-V=v$. Combining this with (5.1) we see

$$
\begin{gathered}
\left.\int_{\mathcal{O}} v(t, \cdot) \psi^{2} d \mu\right|_{t_{1}} ^{t_{2}}+\int_{I} \mathcal{E}_{\mathcal{O}}^{a}\left(v, v ; \psi^{2}\right) d t=-2 \int_{I} \mathcal{E}_{\mathcal{O}}^{a}(v, \psi ; \psi) \\
\leq \frac{1}{2} \int_{I} \mathcal{E}_{\mathcal{O}}^{a}\left(v, v ; \psi^{2}\right) d t+2 c_{1}^{2} \int_{I} \mathcal{E}_{\mathcal{O}}^{a}(\psi, \psi ; 1) d t
\end{gathered}
$$

This implies (7.1).

Lemma 7.2. Let $V(t)=\int v(t, \cdot) \psi^{2} d \mu / \int \psi^{2} d \mu$, where $\psi$ is the function defined after (2.12). Set $c_{29}=2 c_{1}^{2} c_{2} \mu_{2 \gamma} / \gamma^{2}$ and $c_{30}=2 c_{1} c_{4} \gamma^{2}$, then for a.e. $t$

$$
\frac{d V(t)}{d t}+\frac{1}{c_{30} \mu_{\gamma}} \int_{\mathcal{O}(\gamma)}|v(t, x)-V(t)|^{2} d \mu \leq \frac{c_{29}}{\mu_{\gamma}} .
$$

Proof. By (2.9) and the definition of $\psi$, we see $\mathbb{D}^{a}[\psi, \psi] \leq c_{1} c_{2} \gamma^{-2}$. So by (2.1) and Lemma 7.1, we have

$$
\left.\int v \psi^{2} d \mu\right|_{t_{1}} ^{t_{2}}+\frac{1}{2 c_{1}} \int_{t_{1}}^{t_{2}} d t \int \mathbb{D}[v, v] \psi^{2} d \mu \leq c_{29}\left(t_{2}-t_{1}\right) .
$$

Hence, by using the weighted Poincaré inequality (2.12), we obtain

$$
\frac{\left(V\left(t_{2}\right)-V\left(t_{1}\right)\right) \int \psi^{2} d \mu}{t_{2}-t_{1}}+\frac{1}{c_{30}\left(t_{2}-t_{1}\right)} \int_{t_{1}}^{t_{2}} d t \int|v(t, x)-V(t)|^{2} \psi^{2} d \mu \leq c_{29} .
$$

Since $\psi(x)=1$ on $\mathcal{O}(\gamma)$ and $|\psi| \leq 1$, this yields

$$
\frac{\left(V\left(t_{2}\right)-V\left(t_{1}\right)\right) \mu_{\gamma}}{\left(t_{2}-t_{1}\right)}+\frac{1}{c_{30}\left(t_{2}-t_{1}\right)} \int_{t_{1}}^{t_{2}} d t \int_{\mathcal{O}(\gamma)}|v(t, x)-V(t)|^{2} d \mu \leq c_{29} .
$$

Then dividing both sides by $\mu_{\gamma}$ and taking $t_{2} \rightarrow t_{1}$, we obtain (7.2).

Proof of Lemma 3.2. We only prove (3.5) since the proof of (3.4) is similar. Let $c_{31}=$ $\int_{\mathcal{O}(\gamma)} v(0, x) d \mu, c_{32}=c_{29} / \mu_{\gamma}$ and

$$
w(t, x)=v(t, x)-c_{32} t-c_{31}, \quad W(t)=V(t)-c_{32} t-c_{31} .
$$

Then by (7.2) we have

$$
\frac{d W(t)}{d t}+\frac{1}{c_{30} \mu_{\gamma}} \int_{\mathcal{O}(\gamma)}|w(t, x)-W(t)|^{2} d \mu \leq 0 .
$$


For $0<t \leq \gamma^{2}$ let $\mathcal{O}_{s, t}=\{x \in \mathcal{O}(\gamma) ; s<w(t, x)\}$. Then for $s>0$ and $x \in \mathcal{O}_{s, t}$ we have

$$
w(t, x)-W(t)>s-W(t)>0
$$

since $W(t) \leq W(0)=0$ in $0<t \leq \gamma^{2}$, which follows from (7.3). By restricting the integral in (7.3) to $\mathcal{O}_{s, t}$ we obtain

$$
\frac{d W(t)}{d t}+\frac{\mu\left(\mathcal{O}_{s, t}\right)}{c_{30} \mu_{\gamma}}|s-W(t)|^{2} \leq 0,
$$

which immediately implies

$$
\frac{\mu\left(\mathcal{O}_{s, t}\right)}{\mu_{\gamma}} \leq \frac{c_{30}}{(s-W(t))^{2}} \cdot \frac{d(s-W(t))}{d t} .
$$

Integrating from $t=0$ to $t=\gamma^{2}$ and using $W(0)=0$ we see

$$
\frac{1}{\mu_{\gamma}} \bar{\mu}\left(\left\{(t, x) ; 0<t<\gamma^{2}, s<w(t, x)\right\}\right) \leq \frac{c_{30}}{s-W(0)}=\frac{c_{30}}{s} .
$$

This combined with

$$
\begin{aligned}
\bar{\mu}\left(\left\{(t, x) ; 0<t<\gamma^{2}, s<w(t, x)\right\}\right) \\
\quad=\bar{\mu}\left(\left\{(t, x) ; 0<t<\gamma^{2}, s<v(t, x)-c_{32} t-c_{31}\right\}\right) \\
\quad \geq \bar{\mu}\left(\left\{(t, x) ; 0<t<\gamma^{2}, s<v(t, x)-c_{32} \gamma^{2}-c_{31}\right\}\right) \\
\quad=\bar{\mu}\left(\left\{(t, x) ; 0<t<\gamma^{2}, u(t, x)<\exp \left(-s-\left(2 c_{1}^{3} c_{2} \mu_{2 \gamma} / \mu_{\gamma}\right)-c_{31}\right)\right\}\right)
\end{aligned}
$$

completes the proof of (3.5).

\section{Proof of Lemma 3.3}

Let $\sigma$ and $\tau$ be constants such that $1<\sigma, \tau<\infty$. Let $c_{33}=2 c_{1} c_{17}$ and let $c_{34}$ be the constant defined by

$$
c_{34}=\max \left\{\sigma c_{21}\left(r_{2}-r_{1}\right)^{\beta \tau(\sigma-1)}, c_{33}\left(2 c_{16}\right)^{\tau(\sigma-1)}\right\} .
$$

LEMMA 8.1. Let $\varphi(r)=\sup _{Q(r)} \log f$. Then

$$
\varphi\left(r_{1}\right)<\frac{1+\tau}{\sigma \tau} \varphi\left(r_{2}\right)+\frac{c_{34}}{\left(r_{2}-r_{1}\right)^{\beta \tau(\sigma-1)}} \quad \text { for all } \theta \gamma \leq r_{1}<r_{2} \leq \gamma
$$


Proof. Let $\bar{\varphi}=\varphi\left(r_{2}\right) / \sigma$. Decompose $Q\left(r_{2}\right)$ as $Q\left(r_{2}\right)=Q_{+}+Q_{-}$, where $Q_{+}=\left\{x \in Q\left(r_{2}\right) ; \log f>\bar{\varphi}\right\}$ and $Q_{-}=Q\left(r_{2}\right) \backslash Q_{+}$, then by (3.7) we have

$$
\int_{Q\left(r_{2}\right)} f^{p} d \bar{\mu} \leq\left(e^{p \varphi\left(r_{2}\right)} \frac{c_{33}}{\bar{\varphi}}+e^{p \bar{\varphi}}\right) \bar{\mu}(Q(\gamma)) .
$$

We choose $p$ in such a way that the two terms in the brackets on the right-hand side are equal,

$$
p=\frac{1}{\bar{\varphi}(\sigma-1)} \log \left(\frac{\bar{\varphi}}{c_{33}}\right)
$$

provided this quantity is less than $1 / 2 c_{1}$ so that $0<p<1 / 2 c_{1}$ holds. This requires that

$$
\bar{\varphi}>c_{21} .
$$

Assume (8.3) holds, then we have

$$
\int_{Q\left(r_{2}\right)} f^{p} d \bar{\mu} \leq 2 e^{p \bar{\varphi}} \bar{\mu}(Q(\gamma)) .
$$

Combining this with (3.6) yields

$$
\begin{aligned}
\varphi\left(r_{1}\right) & \leq \frac{1}{p} \log \left(\frac{c_{16}}{\left(r_{2}-r_{1}\right)^{\beta}} 2 e^{p \bar{\varphi}}\right) \\
& =\frac{1}{p}\left(\log \frac{2 c_{16}}{\left(r_{2}-r_{1}\right)^{\beta}}\right)+\bar{\varphi} \\
& =\bar{\varphi}\left\{(\sigma-1) \frac{\log \left(\left(2 c_{16}\right) /\left(r_{2}-r_{1}\right)^{\beta}\right)}{\log \left(\bar{\varphi} / c_{33}\right)}+1\right\}
\end{aligned}
$$

by (8.3). Let $1<\tau<\infty$. If the first term in the parentheses is less than $1 / \tau$, that is:

$$
\bar{\varphi}>c_{33}\left(\frac{2 c_{16}}{\left(r_{2}-r_{1}\right)^{\beta}}\right)^{\tau(\sigma-1)},
$$

then we have

$$
\varphi\left(r_{1}\right)<\frac{1+\tau}{\sigma \tau} \varphi\left(r_{2}\right) .
$$

Next assume one of provisions (8.4) and (8.5) is violated, then

$$
\varphi\left(r_{2}\right) \leq \frac{c_{34}}{\left(r_{2}-r_{1}\right)^{\beta \tau(\sigma-1)}} .
$$

Since $\varphi\left(r_{1}\right) \leq \varphi\left(r_{2}\right)$, we obtain (8.1). 
Proof of Lemma 3.3. Let $r_{j}=\gamma(1-(1-\theta) /(1+j))$, then

By (8.1)

$$
r_{j+1}-r_{j}=\frac{\gamma(1-\theta)}{(1+j)(2+j)} \text {. }
$$

$$
\varphi(\gamma \theta) \leq c_{20}\left(\frac{1}{\gamma(1-\theta)}\right)^{\beta \tau(\sigma-1)} \sum_{j=0}^{\infty}\left(\frac{1+\tau}{\sigma \tau}\right)^{j}((1+j)(2+j))^{\beta \tau(\sigma-1)} .
$$

Here $c_{20}=\max \left\{\sigma c_{21}(\gamma(1-\theta))^{\beta \tau(\sigma-1)}, c_{33}\left(2 c_{16}\right)^{\tau(\sigma-1)}\right\}$ as before. Equation (3.8) immediately follows from this.

\section{REFERENCES}

[1] M. T. Barlow and R. F. Bass. Divergence form operators on fractal-like domains. J. Funct. Anal. 175 (2000), 214-247.

[2] M. Fukushima. On a stochastic calculus related to Dirichlet forms and distorted Brownian motions. New stochastic methods in physics. Phys. Rep. 77(3) (1981), 255-262.

[3] M. Fukushima. Distorted Brownian motions and BV functions. Trends in Probability and Related Analysis (Taipei, 1996). World Scientific, Singapore, 1997, pp. 143-150.

[4] M. Fukushima, Y. Oshima and M. Takeda. Dirichlet Forms and Symmetric Markov Processes. Walter de Gruyter, 1994.

[5] A. A. Grigor'yan. The heat equation on noncompact Riemannian manifolds. Math. USSR Sbornik 72-1 (1992), 47-77.

[6] J. Moser. A Harnack inequality for parabolic differential equations. Commun. Pure Appl. Math. 17 (1964), 101-134.

[7] J. Moser. On a pointwise estimate for parabolic differential equations. Commun. Pure Appl. Math. 24 (1971), 727-740.

[8] H. Osada. A family of diffusion processes on Sierpinski carpets. Prob. Theory Relat. Fields 119 (2001), 275-310.

[9] H. Osada. Exotic Brownian motions. Preprint.

[10] L. Saloff-Coste. A note on Poincaré, Sobolev, and Harnack inequalities. Duke J. Math. 65 (1992), 27-32.

[11] K. T. Sturm. Analysis on local Dirichlet spaces III. The parabolic Harnack inequality. J. Math. Pure Appl. 75 (1996), 273-297.

\section{Hirofumi Osada}

Graduate School of Mathematics

Nagoya University

Chikusa-ku

Nagoya 464-8602

Japan

(E-mail: osada@math.nagoya-u.ac.jp) 